\title{
Avaliação do metabolismo oxidativo de ovinos intoxicados por cobre e tratados com tetratiomolibdato associado ou não a vitaminas antioxidantes
}

\author{
Oxidative metabolism in copper poisoning sheep treated with tetrathiomolybdate and/or \\ antioxidative vitamins
}

\author{
Rebeca Alves WEIGEL ${ }^{1}$; Enrico Lippi ORTOLANI ${ }^{1}$; Maria Claudia Araripe SUCUPIRA ${ }^{1}$
}

${ }^{1}$ Departamento de Clínica Médica da Faculdade de Medicina Veterinária e Zootecnia da Universidade de São Paulo, São Paulo-SP

\begin{abstract}
Resumo
Para avaliar o efeito da utilização parenteral das vitaminas $\mathrm{C}$ e/ou E associadas ao quelante de cobre, tetratiomolibdato de amônio (TTM), no tratamento de ovinos com intoxicação cumulativa por cobre (ICC), foram analisados o metabolismo oxidativo, através das concentrações sanguíneas de glutationa reduzida (GSH), séricas de malondialdeído (MDA) e ácido úrico, e a habilidade de redução férrica plasmática (HRFP) desses animais. Foram utilizados 24 ovinos machos, da raça Santa Inês, com peso médio de $25 \mathrm{~kg}$ e distribuídos em quatro tratamentos: TTM, TTM e vitamina C (TTM+VC), TTM e vitamina E (TTM+VE) e TTM e vitaminas C e E (TTM+VCE). O quadro de intoxicação cúprica provocou intensa formação de radicais livres, com redução da concentração de GSH e aumento do MDA, apesar do aumento na capacidade antioxidante plasmática, decorrente do aumento da concentração de ácido úrico e da HRFP. Não foi observado benefício algum da utilização das vitaminas antioxidantes, isoladamente ou associadas, com o tratamento clássico baseado na administração de TTM, na redução do estresse oxidativo.
\end{abstract}

Palavras-chave: Vitamina C. Vitamina E. Intoxicação cumulativa por cobre. Estresse oxidativo. Tetratiomolibdato de amônio (TTM).

\begin{abstract}
The effects of vitamins $\mathrm{C}$ and/or E associated to amonium tetrathiomolybdate (TTM) in the treatment of cumulative copper intoxication (ICC) in sheep were analyzed throughout oxidative metabolism. Reduced glutathione (GSH), malondialdehyde (MDA), uric acid and the ferric reducing ability of plasma (FRAP) concentrations were evaluated. Twenty four male sheep, Santa Ines breed, with $25 \mathrm{~kg} \mathrm{BW}$ was distributed in four treatments: TTM, TTM and vitamin $\mathrm{C}(\mathrm{TTM}+\mathrm{VC})$, TTM with vitamin $\mathrm{E}(\mathrm{TTM}+\mathrm{VE})$ and TTM with vitamins $\mathrm{C}$ and $\mathrm{E}$ (TTM+VCE). The intoxication status caused an intensive formation of free radicals with reduction of the GSH concentration and increase of MDA. Despite the increase in the capacity of plasmatic antioxidant observed with the increase of uric acid and FRAP concentrations, it was not observed any benefit with treatments using oxidant vitamins, isolatedly or together with classic treatment only with TTM.
\end{abstract}

Keywords: Vitamin C. Vitamin E. Copper cumulative poisoning. Oxidative Stress. Amonium tetrathiomolybdate (TTM).

\section{Introdução}

O aumento do número de ovinos no Brasil, principalmente na região sudeste, reflete a importância crescente que esta espécie tem tido, especialmente na última década ${ }^{1}$. Esta evolução responde à maior demanda por leite e, em maior escala, pela carne ovina. Como consequência observa-se o crescimento do sistema intensivo de criação, que, embora proporcione a

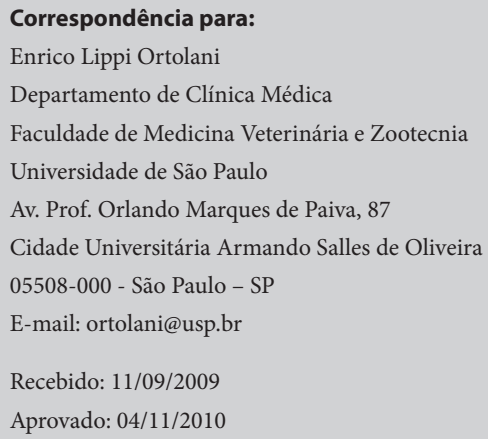


melhora dos índices de produtividade, aumenta a frequência de certas doenças metabólicas, como a urolitíase em machos, toxemia da prenhez em fêmeas e intoxicação por cobre em animais de ambos os sexos².

Geralmente os animais que sucumbem pela intoxicação cumulativa por cobre (ICC), o fazem por insuficiência renal ${ }^{3,4}$, sendo a geração de radicais livres e, portanto o estresse oxidativo uma de suas principais causas. A compreensão do envolvimento do metabolismo oxidativo com a evolução do quadro pode explicar boa parte das lesões encontradas, bem como pode auxiliar na conduta terapêutica, hoje totalmente embasada na exclusiva utilização do quelante de cobre tetratiomolibdato (TTM) nos animais comprometidos.

Entre as espécies de animais domésticos os ovinos são os mais predispostos a apresentarem tanto o quadro de deficiência como o de intoxicação pelo cobre ${ }^{5,6}$. Esta última está relacionada com a menor capacidade de conjugação do cobre com a metalotioneína hepática ${ }^{7}$, diminuindo então a excreção deste elemento do organismo o qual se acumula progressivamente no fígado. Durante essa fase o animal é assintomático, porém quando há saturação de cobre nos hepatócitos, ocorre ruptura destes com liberação de lisossimas e de cobre livre. Este último é um metal monovalente que tem grande tendência a ficar bivalente, interagindo assim com outras substâncias em reações oxidativas. Num sistema biológico, o cobre deverá estar combinado a uma proteína ou a outros compostos e nunca poderá ficar livre, pois neste estado pode provocar danos oxidativos às células ${ }^{8}$, o que acontece quando $o$ animal entra na crise hemolítica $(\mathrm{CH})$.

O tratamento considerado clássico para a intoxicação cúprica foi o preconizado por Gooneratne ${ }^{5}$, que utilizou o tetratiomolibdato (TTM), reconhecido quelante de $\mathrm{Cu}^{+2}$, que é excretado de maneira inerte, favorecendo a sobrevivência dos animais ${ }^{5}$.

O trabalho de Soares ${ }^{4}$ com ovinos intoxicados por cobre demonstrou que até nos animais que sucumbiram existia tentativa do organismo de produzir subs- tâncias antioxidantes endógenas, como foi o caso do aumento dos teores de ácido úrico plasmático, para tentar diminuir a ação nociva dos radicais livres. Porém, este esforço, no caso dos animais não tratados com TTM, foi insuficiente para diminuir os efeitos dos radicais livres no auge da crise hemolítica, sugerindo que teria efeito benéfico à utilização medicamentosa de potentes antioxidantes naturais na melhora do quadro geral, em especial nos danos oxidativos.

Antioxidantes são compostos que protegem sistemas biológicos contra lesões causadas por processos que podem levar a altos níveis de oxidação ${ }^{9}$. Esses compostos são classificados em fatores endógenos, sintetizados no organismo e exógenos, obtidos da $\operatorname{dieta}^{10}$. Este sistema de defesa pode atuar de maneira detoxificadora do agente lesivo antes que ele cause a lesão, ou reparadora da lesão ocorrida ${ }^{11}$. Dentre os antioxidantes endógenos destaca-se a catalase, a glutationa peroxidase (dependente do selênio), a superóxido dismutase (SOD) (dependente de cobre e zinco), o ácido úrico e proteínas ligantes a metais de transição, como transferrina e ceruloplasmina ${ }^{12,13}$. A glutationa reduzida $(\mathrm{GSH})$ atua direta ou indiretamente em processos biológicos importantes e é necessária para prevenir a peroxidação de ácidos graxos insaturados na membrana celular e para manter os átomos de ferro da hemoglobina na forma ferrosa ${ }^{6,14,15}$. O malondialdeído (MDA) é o principal representante do grupo de substâncias reativas ao ácido tiobarbitúrico, originadas da peroxidação de ácidos graxos pelas espécies reativas do oxigênio, tendo suas concentrações aumentadas nos casos de estresse oxidativo ${ }^{16}$. O ácido úrico é considerado um antioxidante não enzimático e atua também formando complexos com o cobre prevenindo a oxidação de substâncias antioxidantes, como a vitamina $\mathrm{C}^{17}$, esta última considerada importante substância antioxidante exógena.

Os antioxidantes exógenos mais comuns são os tocoferóis (vitamina E), ácido ascórbico (vitamina C), carotenoides e flavonoides. Como estes antioxidantes 
estão disponíveis na natureza, suas concentrações podem ser manipuladas através da suplementação e de modificações dietéticas ${ }^{10}$.

Considerando que existem poucos trabalhos que estudam o metabolismo oxidativo de ovinos intoxicados por cobre e não exista trabalho que utilize a associação de antioxidantes neste contexto, esta pesquisa teve por objetivo verificar o potencial benéfico da utilização da vitamina C e/ou da vitamina E associadas ao TTM no tratamento de ovinos acometidos por ICC, através da avaliação física e do metabolismo oxidativo destes animais durante 60 dias a partir da crise hemolítica (hemoglobinúria macroscópica).

\section{Material e Método}

Este experimento está de acordo com os princípios éticos de experimentação animal da Comissão de Bioética da Faculdade de Medicina Veterinária e Zootecnia da Universidade de São Paulo, protocolo número 535/2004.

Foram utilizados 24 ovinos machos, da raça Santa Inês, com cerca de seis meses de idade e aproximadamente $25 \mathrm{~kg}$ de peso vivo (PV). Os animais, provenientes da mesma propriedade, foram mantidos, durante todo o experimento, em gaiolas metabólicas metálicas individuais, providas de cocho e bebedouro. Após a vermifugação, os animais passaram por período de ambientação e adaptação à dieta. Foram considerados adaptados quando mantiveram, por três semanas consecutivas, ganho de peso compatível com a dieta que recebiam.

A intoxicação foi induzida pela administração diária de sulfato de cobre pentahidratado $\left(\mathrm{CuSO}_{4} 5\right.$ $\mathrm{H}_{2} \mathrm{O}, \mathrm{MERCK}^{\oplus}$ ) em solução aquosa por via oral. Foi seguido o modelo descrito por Machado ${ }^{3}$ e Soares ${ }^{4}$. Os animais receberam dose inicial de $3 \mathrm{mg}$ de cobre por $\mathrm{kg}$ de PV por dia (3 mg Cu/kg PV/d) durante uma semana. Não ocorrendo a intoxicação neste período, a dose era acrescida de mais $3 \mathrm{mg} \mathrm{Cu} / \mathrm{kg} \mathrm{PV} / \mathrm{d}$ na pró- xima semana e assim sucessivamente, até a obtenção do quadro de hemoglobinúria macroscópica (HM) ou até atingir a dose de $21 \mathrm{mg} \mathrm{Cu} / \mathrm{kg} / \mathrm{PV} / \mathrm{d}$, que foi fixada até o surgimento da HM. A correção da dose foi realizada de acordo com as pesagens semanais. Os animais eram observados diariamente a fim de se detectar a $\mathrm{CH}$ através da visualização da HM indicada pela urina com coloração amarronzada ("vinho do Porto"). A partir deste momento a administração de cobre foi prontamente interrompida e teve início a coleta de amostras e os animais eram distribuídos em um dos quatro tratamentos: TTM, recebiam TTM, por via intravenosa (IV), na dose de 5,0 mg/kg de Peso Vivo, por quatro dias $(\mathrm{n}=6)$; TTM VC, recebiam o tratamento TTM associado a uma dose de vitamina $\mathrm{C}$ ( $2 \mathrm{~g}$ /animal) pela via intramuscular (IM), esta última administrada 48 horas após a $\mathrm{CH}(\mathrm{n}=6)$; TTM VE, recebiam o tratamento TTM associado à vitamina $\mathrm{E}$, na dose de $200 \mathrm{UI} /$ animal/dia, IM, também por quatro dias $(\mathrm{n}=6)$; TTM VCE, este grupo de animais recebia a associação dos três tratamentos acima descritos $(n=6)$.

O exame físico e a coleta de amostras de sangue e urina foram realizados no momento basal, antes do início da intoxicação (M0), no dia da crise hemolítica (M1), 24 horas após a crise hemolítica (M2), 48 horas após a crise hemolítica (M3), 94 horas após a crise hemolítica (M4), 118 horas após a crise hemolítica (M5), 152 horas após a crise hemolítica, correspondendo a 24 horas após a última dose do tratamento proposto (M6), 10 dias após a crise hemolítica (M7), 15 dias após a crise hemolítica (M8), 30 após a crise hemolítica (M9) e 60 após a crise hemolítica (M10).

Durante o período experimental, os animais foram fisicamente avaliados através da mensuração das funções vitais (frequência cardíaca, frequência respiratória, temperatura retal e movimentos ruminais). Amostras de sangue foram coletadas por punção da veia jugular, em tubos siliconizados vacutainer ${ }^{\circledast}$, 
para a obtenção de soro (sem anticoagulante), plasma (com fluoreto de sódio) e sangue total (com ácido dietileno diamino tetracético - EDTA). Nas amostras de soro foram determinadas as concentrações de malondialdeído (MDA) e ácido úrico pelos métodos descritos por Esterbauer e Cheeseman ${ }^{18}$ e por Fossati, Prencipe e Berti ${ }^{19}$, respectivamente. No plasma foi determinada a habilidade de redução férrica plasmática (HRFP), de acordo com metodologia proposta por Benzie e Strain ${ }^{20}$. A concentração de glutationa reduzida (GSH) foi determinada no sangue total através da técnica descrita por Beutler, Duron e Kelly ${ }^{21}$.

Para a análise das variáveis, foi utilizado o programa estatístico MINITAB RELEASE 13 (2000). A distribuição dos dados foi avaliada inicialmente pelo Teste de Kolgomorov-Smirnov. As variáveis que apresentaram distribuição não-paramétrica foram comparadas pelo teste de Mann-Whitney e as paramétricas pelo teste $t$ de Student. As diferenças foram consideradas significantes quando $\mathrm{P}<0,05$.

\section{Resultados}

$\mathrm{Na}$ avaliação física, com a proximidade da crise hemolítica os ovinos se apresentaram progressivamente apáticos, inapetentes e emaciados. As fezes se tornaram aglomeradas e, posteriormente pastosas com presença de muco. A oligúria ocorreu concomitante com a progressão dos sintomas, culminando com a HM. Com a evolução do quadro os animais perderam peso, período em que ficavam com menor estado de alerta, evoluindo, em alguns casos, para o decúbito esternal. A dose média de cobre (g) para o desencadeamento da $\mathrm{CH}$ foi de 26,10 g no período médio de 61,1 dias.

O perfil das concentrações médias de GSH, MDA, ácido úrico e HRFP estão representados nas tabelas 1, 2,3 e 4 e ilustrados nos gráficos da figura 1.

\section{Discussão}

$\mathrm{Na}$ avaliação do metabolismo oxidativo, as concentrações sanguíneas de GSH, para todos os animais

Tabela 1 - Valores médios e respectivos erros padrão da média (EPM) da concentração sanguínea de glutationa reduzida $(\mathrm{GSH})(\mathrm{mg} / \mathrm{dL})$ de ovinos da raça Santa Inês com intoxicação cúprica cumulativa (ICC) e tratados com tetratiomolibdato (TTM); TTM associado à vitamina $\mathrm{C}(\mathrm{TTM}+\mathrm{VC})$; TTM associado à vitamina $\mathrm{E}(\mathrm{TTM}+\mathrm{VE})$; ou TTM+VCE, antes da crise hemolítica (momento basal - M0); no dia da crise hemolítica ( $\mathrm{CH}-\mathrm{M} 1) ; 24$ h após $\mathrm{CH}(\mathrm{M} 2) ; 48$ h após $\mathrm{CH}$ (M3); 72 h após $\mathrm{CH}(\mathrm{M} 4) ; 96$ h após $\mathrm{CH}$ (M5); 120 h após $\mathrm{CH}$ (M6); 10 dias pós $\mathrm{CH}$ (M7);15 dias pós $\mathrm{CH}$ (M8); 30 dias pós $\mathrm{CH}$ (M9) e 60 dias pós $\mathrm{CH}$ (M10) - São Paulo - 2009

\begin{tabular}{|c|c|c|c|c|c|c|c|c|}
\hline & \multicolumn{2}{|c|}{ TTM } & \multicolumn{2}{|c|}{$T T M+V C$} & \multicolumn{2}{|c|}{ TTM+VE } & \multicolumn{2}{|c|}{ TTM+VEC } \\
\hline MO & $29,61^{\mathrm{abc}}$ & $(4,34)$ & $22,96^{\text {b }}$ & $(5,04)$ & $26,69^{\text {ae }}$ & $(3,94)$ & $25,20^{a}$ & $(1,65)$ \\
\hline M1 & $21,63^{A b c}$ & $(2,93)$ & $15,11^{\mathrm{ABb}}$ & $(2,07)$ & $15,65^{A B c d}$ & $(1,24)$ & $13,23^{\mathrm{Bbcd}}$ & $(2,16)$ \\
\hline M2 & $21,19^{\text {Ace }}$ & $(1,15)$ & $14,14^{\mathrm{Bb}}$ & $(1,80)$ & $13,63^{\mathrm{Bcd}}$ & $(1,14)$ & $13,57^{\mathrm{Bbcd}}$ & $(1,89)$ \\
\hline M3 & $20,32^{c d}$ & $(3,83)$ & $14,96^{b}$ & $(1,72)$ & $15,68^{\mathrm{bcd}}$ & $(1,59)$ & $13,25^{d}$ & $(1,84)$ \\
\hline M4 & $22,55^{\mathrm{cd}}$ & $(2,60)$ & $15,50^{b}$ & $(1,38)$ & $15,05^{\mathrm{bcd}}$ & $(2,20)$ & $16,51^{\text {bcd }}$ & $(1,97)$ \\
\hline M5 & $20,71^{\text {cd }}$ & $(3,39)$ & $18,50^{\mathrm{bc}}$ & $(1,79)$ & $19,55^{\text {bde }}$ & $(1,91)$ & $17,24^{\mathrm{bcd}}$ & $(2,56)$ \\
\hline M6 & $19,29^{\text {ce }}$ & $(1,37)$ & $15,68^{b}$ & $(1,70)$ & $21,18^{\text {bcde }}$ & $(4,41)$ & $19,07^{\text {abd }}$ & $(4,49)$ \\
\hline M7 & $24,70^{\mathrm{ABcd}}$ & $(3,15)$ & $22,65^{\mathrm{Aa}}$ & $(0,50)$ & $18,33^{\text {Bbde }}$ & $(1,46)$ & $26,17^{\mathrm{ABa}}$ & $(4,12)$ \\
\hline M8 & $28,14^{\text {Abde }}$ & $(2,02)$ & $25,13^{\mathrm{ABa}}$ & $(1,66)$ & $21,87^{\text {ABbe }}$ & $(2,53)$ & $20,64^{\mathrm{Bab}}$ & $(1,84)$ \\
\hline M9 & $31,78^{\text {abd }}$ & $(5,37)$ & $27,38^{a}$ & $(3,75)$ & $35,81^{a}$ & $(4,07)$ & $31,04^{a}$ & $(1,13)$ \\
\hline M10 & $59,97^{\mathrm{a}}$ & $(19,50)$ & $36,82^{a c}$ & $(10,40)$ & $39,59^{a}$ & $(2,60)$ & $24,62^{a b d}$ & $(13,20)$ \\
\hline
\end{tabular}

Letras maiúsculas diferentes nas linhas indicam diferenças entre os tratamentos Letras minúsculas diferentes nas colunas indicam diferenças entre os momentos

Nota: Cada grupo experimental era composto de seis animais. Variável analisada pelo teste estatístico ANOVA, com significância para $\mathrm{P} \leq 0,05$ 
Tabela 2 - Valores médios e respectivos erros padrão da média (EPM) da concentração sérica de malondialdeído (MDA) $(\mu \mathrm{mol} / \mathrm{L})$ de ovinos da raça Santa Inês com intoxicação cúprica cumulativa (ICC) e tratados com tetratiomolibdato (TTM); TTM associado à vitamina $\mathrm{C}(\mathrm{TTM}+\mathrm{VC})$; TTM associado à vitamina $\mathrm{E}(\mathrm{TTM}+\mathrm{VE})$; ou TTM+VCE, antes da crise hemolítica (momento basal - M0); no dia da crise hemolítica (CH - M1); 24 h após $\mathrm{CH}$ (M2); 48 h após $\mathrm{CH}$ (M3); $72 \mathrm{~h}$ após $\mathrm{CH}$ (M4); 96 h após CH (M5); 120 h após $\mathrm{CH}$ (M6); 10 dias pós $\mathrm{CH}(\mathrm{M} 7) ; 15$ dias pós $\mathrm{CH}(\mathrm{M} 8) ; 30$ dias pós CH (M9) e 60 dias pós CH (M10) - São Paulo - 2009

\begin{tabular}{|c|c|c|c|c|c|c|c|c|}
\hline & \multicolumn{2}{|c|}{ TTM } & \multicolumn{2}{|c|}{ TTM+VC } & \multicolumn{2}{|c|}{ TTM+VE } & \multicolumn{2}{|c|}{ TTM+VEC } \\
\hline MO & $0,26^{\mathrm{c}}$ & $(0,10)$ & $0,46^{c}$ & $(0,07)$ & $0,38^{c}$ & $(0,05)$ & $0,55^{b}$ & $(0,07)$ \\
\hline M1 & $0,62^{\mathrm{ABa}}$ & $(0,22)$ & $0,98^{A B a c}$ & $(0,19)$ & $1,25^{\mathrm{Aa}}$ & $(0,19)$ & $0,81^{\mathrm{Ba}}$ & $(0,07)$ \\
\hline M2 & $1,02^{a}$ & $(0,23)$ & $1,03^{b}$ & $(0,13)$ & $1,26^{a}$ & $(0,19)$ & $0,90^{\mathrm{a}}$ & $(0,12)$ \\
\hline M3 & $0,96^{a}$ & & $1,07^{\mathrm{ac}}$ & & $1,52^{a b}$ & & $0,96^{\mathrm{ab}}$ & $(0,18)$ \\
\hline M4 & $1,11^{a}$ & $(0,14)$ & $1,28^{a c}$ & $(0,32)$ & $1,86^{a b}$ & $(0,29)$ & $1,10^{a}$ & $(0,2$ \\
\hline M5 & $0,94^{\mathrm{ac}}$ & $(0,45)$ & $1,38^{a c}$ & $(0,38)$ & $1,95^{b}$ & $(0,23)$ & $0,90^{\mathrm{ab}}$ & $(0,19)$ \\
\hline M6 & $0,85^{\mathrm{Ba}}$ & $(0,19)$ & $1,63^{A B a c}$ & $(0,36)$ & $2,02^{\text {Aab }}$ & $(0,41)$ & $1,12^{\mathrm{ABa}}$ & $(0,17)$ \\
\hline M7 & $1,32^{a c}$ & $(0,48)$ & $0,77^{\mathrm{ab}}$ & $(0,13)$ & $2,41^{a b}$ & $(0,85)$ & $1,30^{a}$ & $(0,37)$ \\
\hline M8 & $0,56^{\mathrm{ac}}$ & $(0,22)$ & $0,79^{a b}$ & $(0,18)$ & $1,20^{\mathrm{ac}}$ & $(0,63)$ & $0,69^{a b}$ & $(0,08)$ \\
\hline M9 & $0,49^{\mathrm{cb}}$ & $(0,14)$ & $0,70^{a b}$ & $(0,18)$ & $1,19^{a c}$ & $(0,53)$ & $0,59^{\mathrm{ab}}$ & $(0,25)$ \\
\hline M10 & $1,49^{a c}$ & $(1,11)$ & $0,61^{b}$ & $(0,07)$ & $0,84^{a c}$ & $(0,44)$ & $0,66^{\mathrm{ab}}$ & $(0,36)$ \\
\hline
\end{tabular}

Letras maiúsculas diferentes nas linhas indicam diferenças entre os tratamentos

Letras minúsculas diferentes nas colunas indicam diferenças entre os momentos.

Nota: Cada grupo experimental era composto de seis animais. Variável analisada pelo teste

estatístico ANOVA, com significância para $\mathrm{P} \leq 0,05$

Tabela 3 - Mediana, com respectivos valores mínimos e máximos das concentrações de ácido úrico (mmol/L) de ovinos da raça Santa Inês com intoxicação cúprica cumulativa (ICC) e tratados com tetratiomolibdato (TTM); TTM associado à vitamina C (TTM+VC); TTM associado à vitamina $\mathrm{E}(\mathrm{TTM}+\mathrm{VE})$; ou TTM+VCE, antes da crise hemolítica (momento basal - M0); no dia da crise hemolítica (CH - M1); 24 h após CH (M2); 48 h após CH (M3); $72 \mathrm{~h}$ após $\mathrm{CH}$ (M4); $96 \mathrm{~h}$ após $\mathrm{CH}$ (M5); $120 \mathrm{~h}$ após $\mathrm{CH}$ (M6); 10 dias pós $\mathrm{CH}$ (M7);15 dias pós $\mathrm{CH}$ (M8); 30 dias pós $\mathrm{CH}$ (M9) e 60 dias pós $\mathrm{CH}$ (M10) - São Paulo 2009

\begin{tabular}{|c|c|c|c|c|c|c|c|c|}
\hline \multirow[b]{2}{*}{ MO } & \multicolumn{2}{|c|}{ TाM } & \multicolumn{2}{|c|}{ TTM+VC } & \multicolumn{2}{|c|}{ TTM+VE } & \multicolumn{2}{|c|}{ TTM+VEC } \\
\hline & $0,03^{a}$ & $(0,02-0,23)$ & 0,11 & $(0,09-0,18)$ & 0,09 & $(0,09-0,22)$ & 0,02 & $(0,02-0,11)$ \\
\hline M1 & $0,38^{A b}$ & $(0,1-1,05)$ & $0,71^{A B}$ & $(0,33-2,37)$ & $1,04^{A}$ & $(0,47-8,85)$ & $0,27^{A B}$ & $(0,15-2,28)$ \\
\hline M2 & $0,24^{a b}$ & $(0,05-1,36)$ & $1,23^{A B}$ & $(0,35-5,92)$ & $2,08^{A}$ & $(0,52-7,93)$ & $0,67^{A B}$ & $(0,13-2,01)$ \\
\hline M3 & $0,15^{\mathrm{Bab}}$ & $(0,06-0,4$ & $0,62^{A B}$ & $(0,30-5,13)$ & $1,18^{A}$ & $(0,29-5,00)$ & $0,50^{A B}$ & $(0,06-1,22)$ \\
\hline M4 & $0,07^{\text {Bab }}$ & $(0,05-0,38)$ & $0,79^{A}$ & $(0,33-3,36)$ & $1,13^{A}$ & & $0,28^{A B}$ & $(0,04-1,13)$ \\
\hline M5 & $0,07^{a b}$ & $(0,04-1,32)$ & 0,75 & $(0,27-3,36)$ & 0,68 & $(0,27-3,06)$ & 0,27 & $(0,04-0,77)$ \\
\hline M6 & $0,05^{a b}$ & $(0,03-0,77)$ & 0,29 & $(0,20-1,00)$ & 0,60 & $(0,30-1,86)$ & 0,13 & $(0,03-0,70)$ \\
\hline M7 & $0,05^{a b}$ & $(0,02-0,6$ & 0,32 & $(0,15-0,36)$ & 0,29 & $(0,22-0,43)$ & 0,06 & $(0,03-0,32)$ \\
\hline M8 & $0,03^{a b}$ & $(0,02-0,32)$ & 0,30 & $(0,28-0,32)$ & 0,26 & $(0,09-0,28)$ & 0,30 & $(0,04-1,02)$ \\
\hline M9 & $0,04^{a b}$ & $(0,03-0,30)$ & 0,36 & $(0,15-0,83)$ & 0,20 & $(0,14-0,21)$ & 0,22 & $(0,03-0,42)$ \\
\hline M10 & $0,00^{a b}$ & $(0,00-0,01)$ & 0,27 & $(0,21-0,36)$ & 0,23 & $(0,19-0,26)$ & 0,03 & $(0,03-0,16)$ \\
\hline
\end{tabular}

Letras maiúsculas diferentes nas linhas indicam diferenças entre os tratamentos

Letras minúsculas diferentes nas colunas indicam diferenças entre os momentos.

Nota: Cada grupo experimental era composto de seis animais. Variável analisada pelo teste estatístico Mann-

Whitney, com significância para $\mathrm{P} \leq 0,05$ 
Tabela 4 - Valores médios e respectivos erros padrão da Habilidade de Redução Férrica Plasmática (HRFP) ( $\mu \mathrm{mol} / \mathrm{L})$ de ovinos da raça Santa Inês com intoxicação cúprica cumulativa (ICC) e tratados com tetratiomolibdato (TTM); TTM associado à vitamina $\mathrm{C}(\mathrm{TTM}+\mathrm{VC})$; TTM associado à vitamina E (TTM+VE); ou TTM+VCE, antes da crise hemolítica (momento basal - M0); no dia da crise hemolítica ( $\mathrm{CH}-\mathrm{M} 1) ; 24$ h após $\mathrm{CH}$ (M2); $48 \mathrm{~h}$ após $\mathrm{CH}(\mathrm{M} 3) ; 72 \mathrm{~h}$ após $\mathrm{CH}(\mathrm{M} 4) ; 96 \mathrm{~h}$ após $\mathrm{CH}$ (M5); 120 h após $\mathrm{CH}$ (M6); 10 dias pós $\mathrm{CH}$ (M7);15 dias pós $\mathrm{CH}$ (M8); 30 dias pós CH (M9) e 60 dias pós CH (M10) - São Paulo - 2009

\begin{tabular}{|c|c|c|c|c|c|c|c|c|}
\hline \multirow[b]{2}{*}{ MO } & \multicolumn{2}{|c|}{ TTM } & \multicolumn{2}{|c|}{ TTM+VC } & \multicolumn{2}{|c|}{ TTM+VE } & \multicolumn{2}{|c|}{ TTM+VEC } \\
\hline & $273,32^{c A}$ & $(28,50)$ & $193,73^{\mathrm{Bb}}$ & $(13,50)$ & $238,20^{\mathrm{ABbc}}$ & $(26,90)$ & $277,01^{\mathrm{Aa}}$ & $(32,70)$ \\
\hline M1 & $3182,40^{\text {ac }}$ & $(1608,00)$ & $2439,45^{a b}$ & $(1188,00)$ & $3684,22^{\text {acd }}$ & $(1286,00)$ & $3594,00^{\mathrm{bcd}}$ & $(1255,00)$ \\
\hline M2 & $2645,92^{a}$ & $(1375,00)$ & $3076,76^{a b}$ & $(1640,00)$ & $5147,81^{\text {ab }}$ & $(2164,00)$ & $9836,77^{c}$ & $(3329,00)$ \\
\hline M3 & $1320,08^{\mathrm{Ba}}$ & $(450,00)$ & $4796,17^{\mathrm{ABab}}$ & $(2676,00)$ & $2805,13^{\mathrm{Bab}}$ & $(1217,00)$ & $8629,86^{\mathrm{Ad}}$ & $(2030,00)$ \\
\hline M4 & $568,30^{\text {a }}$ & $(80,00)$ & $3452,18^{a b}$ & $(1816,00)$ & $3673,02^{a b}$ & $(1515,00)$ & $3509,82^{\text {abcd }}$ & $(2042,00)$ \\
\hline M5 & $569,18^{a}$ & $(47,40)$ & $2930,52^{a b}$ & $(1489,00)$ & $3121,08^{a b}$ & $(1251,00)$ & $3880,24^{b c d}$ & $(1777,00)$ \\
\hline M6 & $1056,48^{a b c}$ & $(505,00)$ & $1338,28^{a b}$ & $(827,00)$ & $3738,95^{a b}$ & $(1454,00)$ & $786,32^{b}$ & $(195,00)$ \\
\hline M7 & $409,30^{a b c}$ & $(77,40)$ & $374,28^{a}$ & $(58,70)$ & $435,83^{b}$ & $(136,00)$ & $577,12^{b}$ & $(93,70)$ \\
\hline M8 & $294,14^{A B b}$ & $(32,50)$ & $210,83^{\text {Bab }}$ & $(6,29)$ & $324,65^{\mathrm{ABc}}$ & $(44,00)$ & $512,07^{A b c}$ & $(103,00)$ \\
\hline M9 & $223,53^{\mathrm{Bbc}}$ & $(11,70)$ & $260,53^{A B a}$ & $(26,00)$ & $170,02^{\mathrm{Bd}}$ & $(20,80)$ & 392,20 Aac & $(70,10)$ \\
\hline M10 & $206,76^{b c}$ & $(42,60)$ & $221,60^{\text {ab }}$ & $(68,10)$ & $169,72^{\mathrm{bcd}}$ & $(34,30)$ & $246,60^{\text {ac }}$ & $(23,70)$ \\
\hline
\end{tabular}

Letras maiúsculas diferentes nas linhas indicam diferenças entre os tratamentos Letras minúsculas diferentes nas colunas indicam diferenças entre os momentos

Nota: Cada grupo experimental era composto de seis animais. Variável analisada pelo teste estatístico ANOVA, com significância para $\mathrm{P} \leq 0,05$
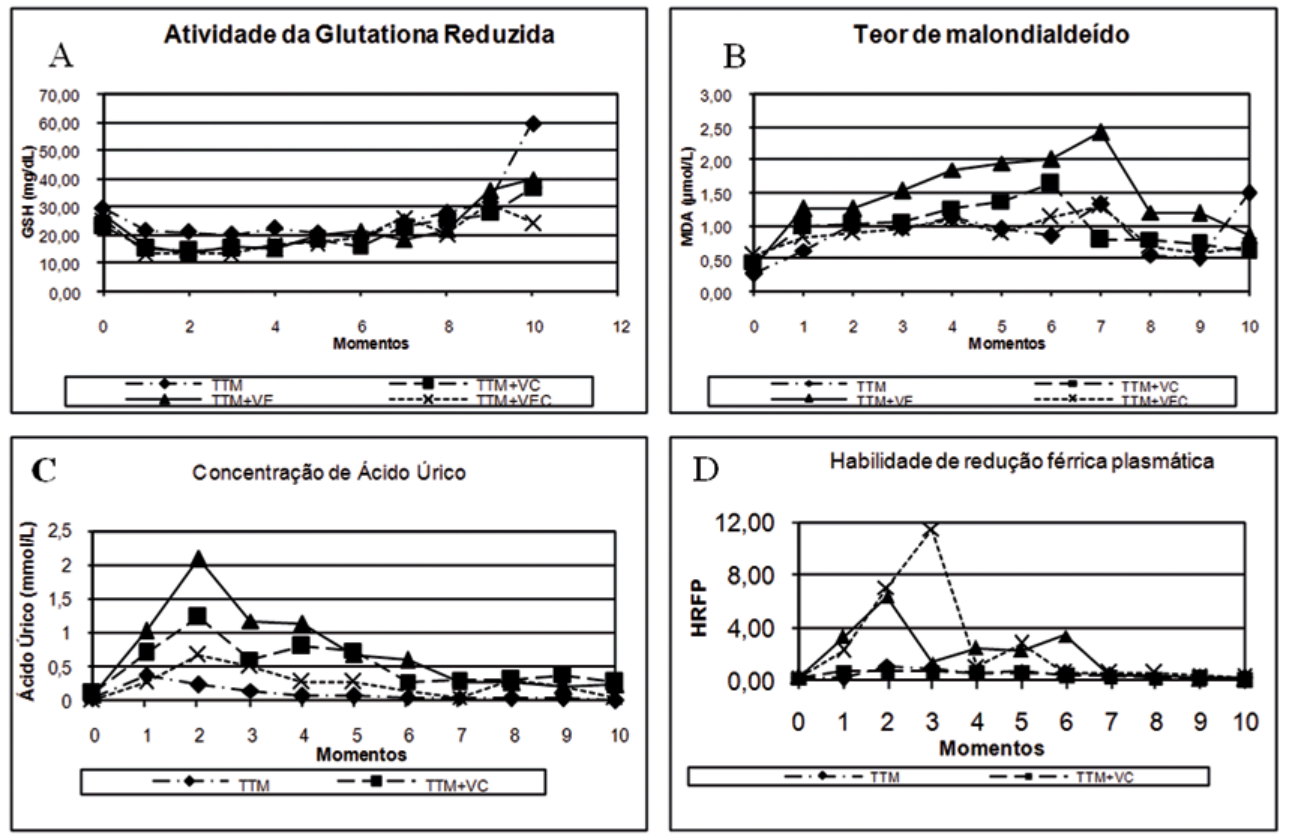

Figura 1 - Perfil dos valores médios da concentração sanguínea de GSH(mg/dL) (A); concentração sérica de MDA ( $\mu \mathrm{mol} / \mathrm{L})(\mathrm{B})$; concentração sérica de ácido úrico $(\mathrm{mmol} / \mathrm{L})(\mathrm{C})$; e valores plasmáticos da $\operatorname{HRFP}(\mu \mathrm{mol} / \mathrm{L})(\mathrm{D})$ de ovinos da raça Santa Inês com intoxicação cúprica cumulativa (ICC) e tratados com tetratiomolibdato (TTM); TTM associado à vitamina $\mathrm{C}(\mathrm{TTM}+\mathrm{VC})$; TTM associado à vitamina $\mathrm{E}$ (TTM+VE); ou TTM+VCE, antes da crise hemolítica (momento basal - M0); no dia da crise hemolítica ( $\mathrm{CH}-\mathrm{M} 1) ; 24$ h após $\mathrm{CH}$ (M2); 48 h após $\mathrm{CH}$ (M3); $72 \mathrm{~h}$ após $\mathrm{CH}(\mathrm{M} 4) ; 96$ h após $\mathrm{CH}$ (M5); 120 h após $\mathrm{CH}$ (M6); 10 dias pós $\mathrm{CH}$ (M7);15 dias pós $\mathrm{CH}$ (M8); 30 dias pós $\mathrm{CH}$ (M9) e 60 dias pós $\mathrm{CH}$ (M10) 
submetidos aos quatro tratamentos, foram menores na $\mathrm{CH}$ que 60 dias após (M10). Interessante observar que, diferente dos ovinos pertencentes ao tratamento TTM+VCE, que tiveram concentrações semelhantes, os animais submetidos aos outros três tratamentos tiveram concentrações de glutationa reduzida (GSH) maiores em M10 que no M0. Deve ser ressaltado, porém, que os 24 animais utilizados apresentaram, no momento basal, baixa concentração média de glutationa reduzida $(26,12 \mathrm{mg} / 100 \mathrm{~mL})$. Os valores de GSH considerados adequados estão acima de $40 \mathrm{mg} / 100$ $\mathrm{mL}^{22}$, isto pode significar que parte dos ovinos utilizados neste experimento tinham problema genético responsável pela deficiência no transporte de certos aminoácidos, resultando em baixas concentrações de GSH e resultando na menor capacidade antioxidante proporcionada por este tripeptídeo ${ }^{23}$. $\mathrm{Na}$ comparação entre os tratamentos, as concentrações de GSH em quatro momentos foram superiores para os animais tratados com TTM em relação aos tratados com TTM+VCE (Tabela 1).

Diferente do GSH, a concentração de MDA no momento basal foi menor que na $\mathrm{CH}$ e nos dez dias seguintes. Os maiores valores de MDA no grupo tratado apenas com a vitamina $\mathrm{E}$ podem ser explicados pela alta dose utilizada no presente estudo. Brigelius-Flohé e Traber ${ }^{24}$ apontaram que, em certas situações, esta vitamina pode ter também função pró-oxidante, sendo que esta condição dificilmente ocorre na presença de outras substâncias antioxidantes, como a vitamina C. No atual estudo, embora um dos tratamentos se va-

\section{Referências}

1.BARBOSA, J. A. Especialista recomenda investimento em ovinos. Disponível em: <http://www.ovinosecia.com.br >. Acesso em: 2003

2. ORTOLANI, E. L. Intoxicações metabólicas em ovinos: intoxicação cúprica. In: SILVA SOBRINHO, A. G.; BATISTA, A. M. V.; SIQUEIRA, E. R.; ORTOLANI, E. L.; SUSIN, I.; SILVA, J. I. C.; TEIXEIRA, J. C.; BORBA, M. F. S. Nutrição de ovinos. Jaboticabal: Funep, 1996. p. 241-246.

3. MACHADO, C. H. Uso de tetratiomolibdato no tratamento lesse da associação das vitaminas $\mathrm{C}$ e $\mathrm{E}$, a primeira era administrada somente 48 horas após a $\mathrm{CH}$, fato que permitiu a possível ação pró-oxidante da vitamina $\mathrm{E}$.

Os dados relativos à HRFP e às concentrações de ácido úrico indicaram maiores concentrações destas durante a crise hemolítica (Tabelas 3 e 4). Estes dois marcadores da capacidade antioxidante demonstraram que houve resposta compensatória frente à grande formação de radicais livres durante o período mais próximo à $\mathrm{CH}$, como também foi observado por Soares ${ }^{4}$. O ácido úrico em concentrações elevadas indica a intensificação do metabolismo oxidativo no organismo, o que foi bem ilustrado neste estudo.

\section{Conclusão}

Pelo comportamento destes quatro indicadores do status oxidativo, é possível observar que, principalmente durante os primeiros dez dias da crise hemolítica, em todos os tratamentos, houve diminuição das concentrações de GSH e aumento de MDA e, na tentativa de coibir o estresse oxidativo, ocorreu aumento com posterior decréscimo de ácido úrico e de HRFP. O comportamento destas variáveis foi reflexo da intoxicação cumulativa por cobre, que causou aos ovinos utilizados um grande incremento no metabolismo oxidativo. Embora tenham ocorrido diferenças pontuais nestes indicadores, entre os tratamentos utilizados, não foi observado benefício da associação das vitaminas antioxidantes $\mathrm{C}$ e/ou $\mathrm{E}$, no controle dos radicais livres no atual estudo.

de intoxicação cúprica experimental em ovinos: avaliações clínica e toxicológica. São Paulo, 1998, 138 f. Tese (Doutorado em Clínica Veterinária) - Faculdade de Medicina Veterinária e Zootecnia, Universidade de São Paulo, São Paulo, 1998.

4. SOARES, P. C. Efeito da intoxicação cúprica e do tratamento com tetratiomolibidato sobre a função renal e o metabolismo oxidativo de ovinos. 2004. 117 f. Tese (Doutorado em Clínica Veterinária) - Faculdade de Medicina Veterinária e Zootecnia, Universidade de São Paulo, São Paulo, 2004. 
5. GOONERATNE, S. R.; HOWELL, J. M.; KUMARATILAKE, J. $\mathrm{S}$. Kidney function in copper toxicity in sheep. In: International symposium on trace element metabolism in man and animals. Australian Academy of Science, v. 46, p. 461-464. 1981.

6.SANSINANEA, A. S.; CERONE, S. I.; ELPERDING, A.; AUZA, N. Glucose-6-phosphate dehydrogenase activity in eruthrocyts from chronically copper poisoned sheep. Comparative Biochemistry and Physiology, v. 114, n. 3, p. 197-200, 1996

7. BREMNER, I. Involvement of metallothionein in the hepatic metabolism of copper. Journal of Nutrition, v. 117, n. 1, p. 19-29, 1987.

8. ORTOLANI, E. L.; MACHADO, C. H.; SUCUPIRA, M. C. A. Assessment of some clinical and laboratory variables for early diagnosis of accumulative copper poisoning in sheep. Veterinary \& Human Toxicology, v. 45, n. 6, p. 289-293, 2003.

9. KRINSKI, N. I. Mechanism of action of biological antioxidants. Proceedings of the Society for Experimental Biology and Medicine, v. 200, n. 2, p. 248-254, 1992.

10.GALIZIA, M. S.; WAITZBERG, D. L. Mecanismo de ação dos radicais livres e antioxidantes. Revista Brasileira de Nutrição Clínica, v. 16, n. 2, p. 79-89, 2001.

11.FERREIRA, A. L. A.; MATSUBARA, L. S. Radicais livres: conceitos, doenças relacionadas, sistemas de defesa e estresse oxidativo. Revista da Associação Médica Brasileira, v. 43, n. 1, p. 61-68, 1997.

12.HALLIWELL, B.; CHIRICO, S. Lipid peroxidation: its mechanism, measurement and significance. American Journal of Clinical Nutrition, v. 57, p. 715S-725S, 1993. Supplement, 5 .

13. HALLIWELL, B. Mechanisms involved in the generation of free radicals. Pathology and Biology, v. 44, n. 1, p. 6-13, 1996.

14. ROVER JÚNIOR, L.; HÖEHR, N. F.; VELLASCO, A. P.; KUBOTA, L. T. Sistema antioxidante envolvendo o ciclo metabólico da glutationa associada a métodos eletroanalíticos na avaliação do estresse oxidativo. Química Nova, v. 24, n. 1, p. 112-119, 2001.

15.WU, G.; FANG, Y. Z.; YANG, S.; LUPTON, J. R.; TURNER, N. D. Glutathione metabolism and its implications for health. Journal of Nutrition, v. 134, n. 3, p. 488-492, 2004.

16. LIMA, E. S.; ABDALLA, D. S. P. Peroxidação lipídica: mecanismos e avaliação em amostras biológicas. Brazilian Journal of Pharmaceutical Sciences, v. 37, n. 3, p. 293-303, 2001.

17. CHIHUAILAF, R. H.; CONTRERAS, P. A.; WITTWER, F. G. Pathogenesis of oxidative stress: consequences and evaluation in animal health. Veterinaria México, v. 33, n. 3, p. 265-283, 2002.

18.ESTERBAUER, H.; CHEESEMAN, K. H. Determination of aldehydic lipid peroxidation products: malonaldeyde and 4-hydroxynonenal. Methods in Enzymology, v. 186, p. 407$421,1990$.

19. FOSSATI, P.; PRENCIPE, L.; BERTI, G. Use of 3,5-dichloro-2hydroxybenzenesulfonic acid/4-aminophenazone chromogenic system in direct enzymic assay of uric acid in serum and urine. Clinica Chemistry, v. 26, n. 2, p. 227-231, 1980.

20.BENZIE, I. F. F.; STRAIN, J. J. The ferric reducing ability of plasm (FRAP) as a measure of antioxidant power: The FRAP assay. Analytical Biochemistry, v. 239, n. 1, p. 70-76, 1996.

21.BEUTLER, E.; DURON, O.; KELLY, B. M. Improved method for the determination of blood glutatione. Journal Laboratory Clinical Medicine, v. 61, n. 5, p. 882-888, 1963.

22.MARONPOT, R. R. Erytrocyte glucose-6-phosphate dehydrogenase and glutatione deficiency in sheep. Canadian Journal of Comparative Medicine and Veterinary Science, $v$. 36, n. 1, p. 55-60, 1972.

23. YONG, J. D.; TUCKER, E. M.; KILGOUR, L. Genetic control of aminoacid transport in sheep erythrocytes. Biochemical Genetics, v. 20, n. 7-8, p. 723-731, 1982.

24.BRIGELIUS-FLOHÉ, R.; TRABER, M. G. Vitamin E: function and metabolism. The FASEB Journal, v. 13, n. 10, p. 11451155,1999 